\title{
Iron homeostasis in plants: when transcription affects translocation
}

\author{
Henri Wintz ${ }^{1}$ \\ ${ }^{1}$ Department of Nutritional Sciences and Toxicology, University of California, Berkeley, CA 94720, USA
}

Cell Research (2006) 16:797-798. doi:10.1038/sj.cr.7310102; published online 16 October 2006

\begin{abstract}
Despite the fact that iron is one of the most abundant elements of the earth's crust, iron deficiencies are serious problems both in human nutrition [1] and in agriculture [2]. Six to eight percent of the world's population is potentially affected by iron deficiency induced anemia, a leading cause of maternal death in African and Asian countries where people rely mostly on plants for their daily intake of iron. Iron can also be a limiting factor in the growth of economically important crop plants because of inadequate soil chemistry, and such deficiencies cannot easily be corrected by amending the soil. Improving the plant's ability to absorb iron in adverse conditions and to increase their overall content could offer solutions to these dramatic problems. Therefore understanding the molecular mechanisms regulating iron uptake and homeostasis in plants has potentially important practical applications both in agriculture and human health [3].
\end{abstract}

Great strides have been made in recent years in identifying key genes and proteins involved in iron transport and in demonstrating the importance of transcriptional regulation of these genes in response to the iron status of the plants [4]. It was demonstrated that long distance signaling from the shoot to the roots is key to regulating iron uptake in the roots [5]. However, there is still a significant gap and virtually no progress has been made in our understanding on how plants sense their iron status and how the signals are transduced across the plant toward the root cells to induce or repress the expression of key genes involved in iron homeostasis such as the genes coding for ferric reductases (FRO) or the iron transporters (IRT1, IRT2).

As pointed out by Chen et al in their article of this issue of Cell Research [6], there are only two accounts in the literature describing transcription factors involved in the regulation of iron metabolism in plants [7, 8]. Therefore the work reported by Chen et al is of significant importance in the quest to identifying key regulatory genes involved in iron homeostasis. Chen et al discovered that a transcription factor of the R2R3 Myb family (DwMYB2) isolated from an orchid (Dendrobium hybrid) caused iron deficiency-like symptoms when expressed in the model plant Arabidopsis thaliana. They demonstrated that overexpression of DwMYB2 led to gene expression profiles characteristic of iron deficiency that were likely caused by an imbalance in the distribution of iron between roots and shoots, and concluded that, when expressed in Arabidopsis this transcription factor affects iron translocation between the roots and the shoots.

The effect of the heterologous MYB gene expression on iron homeostasis could be either by direct induction of the expression of genes or by deregulation via competitive interaction with endogenous transcription factor binding sites. A detailed analysis of gene expression profiles using a full genome array will help identify the direct or indirect targets of this heterologous transcription factor and open avenues of investigations to further understand the cascade of events involved in the affected regulatory pathways in Arabidopsis. Analysis of the tissue expression pattern of this gene in orchids will help understand the native role of this transcription factor. Expression in tissues associated with long distance transport of nutrients and potential regulation in response to the iron status would provide evidence for a native role of DwMYB2 in metal transport in orchids. Because there is no closely related ortholog of the orchid gene in Arabidopsis, the direct role of the gene in controlling the expression of genes involved in iron homeostasis could not be examined by analyzing a loss-of-function mutant. The next task will be to identify which of the 125 known R2R3 Myb transcription factors in Arabidopsis [9] is involved in iron metabolism regulation. The use of genomics resources and approaches such as knockout mutants, overexpressors and gene expression profiling will be crucial to undertake this task. 


\section{References}

1 WHO. (World Health Organization,Department of Nutrition for Health and Development, Geneva, Switzerland, 2004).

2 Guerinot ML. Improving rice yields--ironing out the details. Nat Biotechnol 2001; 19:417-418.

3 Guerinot ML. Perspectives: plant biology. The green revolution strikes gold. Science 2000; 287:241-243.

4 Wintz H, Fox T, Wu YY, et al. Expression profiles of Arabidopsis thaliana in mineral deficiencies reveal novel transporters involved in metal homeostasis. J Biol Chem 2003; 278:47644-47653.

5 Grusak MA, Pezeshgi S. Shoot-to-Root signal transmission regulates root Fe(III) reductase activity in the dgl mutant of Pea. Plant Physiol 1996; 110:329-334.

6 Chen YH, Wu XM, Ling HQ, Yang WC. Transgenic expression of DwMYB2 impairs iron transport from root to shoot in Arabidopsis thaliana. Cell Res 2006; 16:830-840

7 Ling HQ, Bauer P, Bereczky Z, Keller B, Ganal M. The tomato fer gene encoding a bHLH protein controls iron-uptake responses in roots. Proc Natl Acad Sci USA 2002; 99:13938-13943.

8 Yuan YX, Zhang, J, Wang, DW, Ling, HQ. AtbHLH29 of Arabidopsis thaliana is a functional ortholog of tomato FER involved in controlling iron acquisition in strategy I plants. Cell Res 2005; 15:613-21.

9 Stracke R, Werber M, Weisshaar B. The R2R3-MYB gene family in Arabidopsis thaliana. Curr Opin Plant Biol 2001; 4:447-456. 